\title{
Isotretinoin unter falschem Verdacht?
}

\author{
Wie groß ist die Gefahr, unter einer Therapie mit Isotretinoin Depressionen zu entwickeln, \\ wirklich? Einer Metaanalyse aus Taiwan zufolge wird diese Nebenwirkung des Aknemedikaments \\ möglicherweise überschätzt.
}



Dass Isotretinoin bei den damit behandelten Aknepatienten möglicherweise Depressionen auslösen kann, wurde erstmals 1983 beschrieben. 1998 hat die US-Arzneimittelbehörde FDA (Food and Drug Administration) eine diesbezügliche Warnung herausgegeben. Seither sind zum Thema immer wieder (nicht randomisierte) Studien publiziert worden, die jedoch widersprüchliche Ergebnisse lieferten und vor allem nie einen kausalen Zusammenhang belegen konnten.

\section{Metaanalyse mit 31 Studien}

Ein Team der Taipei Medical University in Taiwan hat nun eine umfassende Literaturauswertung vorgelegt, in der 31 Studien aus dem gesamten Zeitraum seit Einführung der Isotretinointherapie bis Ende 2016 berücksichtigt wurden. In die Metaanalyse flossen 8 kontrollierte, 20 prospektive Open-label- und 3 bevölkerungsbasierte Studien ein.

Das überraschende Ergebnis der Literaturauswertung: Zwischen der Isotretinoineinnahme und der Entwicklung von Depressionen fand sich in der Zusammenschau kein signifikanter Zusammenhang. In beinahe der Hälfte der Studien (11 von 25, davon 2 kontrolliert und 9 open-label) hatten sich bestehende depressive Symptome sogar deutlich gebessert oder in ihrer Frequenz verringert.

In 6 kontrollierten Studien entwickelten sich die Depressions-Scores unter den verglichenen Therapieregimen (Isotretinoin versus eine alternative Therapie, zum Beispiel Antibiotika) nahezu gleich, mit einer standardisierten mittleren Abweichung („standardized mean difference“, SMD) von -0,33 (95\%-KI -0,680 bis 0,011). Beide Regime führten zu einer Verbesserung der Symptome.
Nach Beginn einer Isotretinointherapie sank die Prävalenz der Depression insgesamt signifikant, berichten Dr. Yu-Chen Huang und sein Forscherteam; das relative Risiko betrug 0,588 (95\%-KI 0,382 bis 0,904).

\section{Depressionswerte gehen zurück}

In einer Analyse, die kontrollierte und nicht kontrollierte Studien zusammenfasste, gingen nach Verabreichung von Isotretinoin die mittleren Werte in den verschiedenen Skalen für Depression (Beck Depression Inventory, Hospital Anxiety Depression Scale) signifikant zurück (SMD -0,335; 95\%-KI $-0,498$ bis $-0,172$ ).

Die Symptombesserung war sowohl nach den ersten 1 bis 2 Monaten der Behandlung sowie nach 4 Monaten deutlich. In der Spanne zwischen 3 und 4 Monaten nach Therapiebeginn war zumindest ein Trend zu einer Besserung zu erkennen. Wie Huang und Kollegen betonen, zeigte sich innerhalb des für die Aknebehandlung typischerweise eingesetzten Dosisbereichs zwischen 0,5 und $1 \mathrm{mg} / \mathrm{kg}$ pro Tag keine dosisabhängige Risikoerhöhung.

Lediglich ein Forscherteam war zu dem Schluss gekommen, dass Isotretinoin das Depressionsrisiko erhöht hatte. Dabei handelte es sich um eine Bevölkerungsstudie. Darüber hinaus wurde in 2 Open-label-Studien ein Anstieg der Depressionswerte beobachtet, in einer der beiden war das Ergebnis jedoch nicht signifikant.

\section{„Für sich genommen kein Risikofaktor"}

„Isotretinoin für sich genommen scheint kein Risikofaktor für die Entwicklung einer Depression zu sein“, so das Resümee von Huang und Kollegen. Dies gelte zumindest für die genannte therapeutische Dosis zur Behandlung der Akne. Erhöhte Depressionswerte oder neu aufgetretene Fälle in den berücksichtigten Studien stünden möglicherweise in Verbindung mit der psychisch oft sehr belastenden Hauterkrankung selbst. Dies müsse man als behandelnder Arzt berücksichtigen. Die Aknepatienten in den verschiedenen Studien hatten nach Huang und Kollegen oftmals bereits zu Therapiebeginn hohe Werte in Depressionsskalen gezeigt. In 4 Studien hätten diese den Grenzwert für eine manifeste Depression überschritten.

Dennoch wird vor zu hohen Dosen des potenten Aknemedikaments gewarnt: Isotretinoin ist als fettlösliche Substanz in der Lage, die Blut-Hirn-Schranke zu überwinden. Es gibt Studien, die nachweisen, dass das Therapeutikum in dopaminerge und serotonerge Systeme eingreifen sowie die Neurogenese im Hippocampus beeinflussen kann.

Dr. Elke Oberhofer

\section{Literatur}

Yu-Chen Huang et al. J Am Acad Dermatol 2017;76:1068-76.e9 